\title{
JLAMP: AN AMPLIFIER-BASED FEL IN THE JLAB SRF ERL DRIVER*
}

\author{
Kevin Jordan, Stephen V. Benson, David Douglas ${ }^{\#}$, Pavel Evtushenko, Carlos Hernandez-Garcia, \\ George R. Neil, Jefferson Lab, Newport News, VA 23606, U.S.A.
}

\section{Abstract}

Notional designs for energy-recovering linac ("ERL") driven high average power free electron lasers ("FEL"s) often invoke amplifier-based architectures. To date, however, amplifier FELs have been limited in average power output to values several orders of magnitude lower than those demonstrated in optical-resonator based systems; this is due at least in part to the limited electron beam powers available from their driver accelerators. In order to directly contrast the performance available from amplifiers to that provided by high-power cavity-based resonators, we have developed a scheme to test an amplifier FEL in the JLab SRF ERL driver. We describe an accelerator system design that can seamlessly and noninvasively integrate a $10 \mathrm{~m}$ wiggler into the existing system and which provides, at least in principle, performance that would support high-efficiency lasing in an amplifier configuration. Details of the design and an accelerator performance analysis will be presented.

\section{MOTIVATION}

Recent advances in high power FEL performance were achieved through the use of superconducting radiofrequency ("SRF") ERL driver accelerators, and are restricted to cavity-oscillator based systems [1]. In contrast - due to the lack of demonstration experiments amplifier FEL operation at high power is at the state of development that oscillators were eight years prior to the JLab IR Demo [2]. While codes predict reasonable performance, many issues associated with high power operation remain untested, including: maximum saturation efficiency, optical mode quality, limits of optical guiding at saturation, wakefield and resistive wall effects in a long wiggler, limits to control of output divergence, energy spread induced on the drive electron beam for a given FEL efficiency, and harmonic intensity (in comparison to that in oscillators). Further, as no amplifier has yet been tested in an ERL, there is - in addition to these numerous FEL technology questions - virtually no experience base to guide either the design or operation of an integrated high power ERL/amplifier FEL system.

In order to address these issues, we have investigated the integration of an amplifier FEL into the JLab FEL Upgrade [3]. The focus of this study is three-fold: to establish a self-consistent amplifier FEL design, and thence derive drive-beam requirements; to ascertain if an amplifier - with its necessarily long wiggler - could be incorporated with the JLab Upgrade driver accelerator,

\footnotetext{
*This work supported by the Off. of Naval Research, the Joint Technology Off., the Commonwealth of Virginia, the Air Force Research Lab, Army Night Vision Lab, and by DOE Contract DEAC05-060R23177.

\#douglas@jlab.org
}

and to devise a program of machine characterization and development so as to insure any needed beam quality improvements can be provided.

\section{FEL DESIGN AND DRIVE ELECTRON BEAM REQUIREMENTS}

A critical design metric for an FEL amplifier is the gain length. The gain length must be as short as possible and the device must have at least 10 gain lengths to provide meaningful amplification. There are, however other practical aspects that must be taken into account in the design. The wiggler gap has to be reasonably large so that beam loss does not destroy the wiggler and resistive wall heating does not overheat the vacuum chamber. One must find the best compromise between the desire for a large gap in the wiggler and a short gain length. We have found that a good compromise for a $120 \mathrm{MeV}$ electron beam is a $3.3 \mathrm{~cm}$ period with an $11 \mathrm{~mm}$ gap, which is close to the undulator A used at Argonne. Having chosen the wiggler one then has to obtain the brightest electron beam possible. Calculations show that the gain length varies approximately as the square root of both the transverse and longitudinal emittance so improvements in either are equally useful. Since emittances grow with charge we find that the gain length is a very weak function of charge for any given machine. If the charge is greater than about $0.25 \mathrm{nC}$ the gain length is reasonable $(<60 \mathrm{~cm})$. The predicted gain length must be corrected for 3D effects, slippage, and wiggler segmentation if there is any. All these effects tend to increase the gain length.

With a $60 \mathrm{~cm}$ gain length we need at least 6 meters of undulator. If we use two undulator As and an undulator A prototype already at Jefferson Lab we have 6.8 meters of undulators. This would require quadrupoles and phase matching magnets to be mounted between the undulators in the same fashion as the LEUTL FEL [4]. The overall length will be close to 8 meters in length. The vacuum chamber for this is quite a challenge since it has to expand and contract by a few percent due to resistive wall heating. The inside of the chamber must be copper plated and it must have high resolution beam position and beam imaging diagnostics along its length. The chamber must be effectively water cooled to stabilize it length as much as possible.

Another possibility is to use the NISUS wiggler now being employed at the Brookhaven SDL [5]. This is a 10 meter wiggler with a $3.9 \mathrm{~cm}$ period. The gap would have to be closed down from its present value and the gain length is a bit longer than for the $3.3 \mathrm{~cm}$ period wiggler but the greater length and integrated two-plane focusing make this an attractive option. The gap is also larger, which reduces risk and resistive wall heating. 
Table 1 contrasts amplifier drive beam requirements to performance regularly achieved during operation of the JLab IR Upgrade FEL.

Table 1: Electron beam parameters at the wiggler

\begin{tabular}{|l|l|l|}
\hline Parameter & $\begin{array}{l}\text { Amplifier } \\
\text { Requirement }\end{array}$ & $\begin{array}{l}\text { IR Upgrade } \\
\text { Operational }\end{array}$ \\
\hline Beam energy & $120 \mathrm{MeV}$ & $120 \mathrm{MeV}$ \\
\hline Bunch charge & $>250 \mathrm{pC}$ & $135 \mathrm{pC}$ \\
\hline $\begin{array}{l}\text { Normalized } \\
\text { transverse } \\
\text { emittance }\end{array}$ & $<11 \mathrm{~mm}-\mathrm{mrad}$ & $10 \mathrm{~mm}-\mathrm{mrad}$ \\
\hline $\begin{array}{l}\text { Normalized } \\
\text { longitudinal } \\
\text { emittance }\end{array}$ & $<90 \mathrm{keV}$-psec & $90 \mathrm{keV}-\mathrm{psec}$ \\
\hline Wiggler $\mathrm{K}_{\mathrm{rms}}$ & 1.58 & $0.5-2.8$ \\
\hline Wiggler gap & $>11 \mathrm{~mm}$ & $16.5 \mathrm{~mm}$ \\
\hline Energy spread & $<1 / 4 \% \mathrm{rms}$ & $<1 / 2 \% \mathrm{rms}$ \\
\hline
\end{tabular}

\section{DRIVER ACCELERATOR/AMPLIFIER INTEGRATION}

The primary issues for our accelerator design study were: the integration of a long wiggler into a relatively compact ERL; delivery to this wiggler of a properly configured drive beam (as described above); and the recovery of the exhaust drive beam after lasing, without excessive beam loss. The JLab IR Upgrade driver accelerator design includes a bypass beamline parallel to the existing IR FEL [6]. Originally intended for a cavityresonator UV FEL, this beamline (which is partially installed), provides a natural location for the - literally side-by-side - comparison of an amplifier to an oscillator.

To establish an existence-proof solution, we have developed a beam-optics design [7] for an ERL-driven amplifier based on the NISUS wiggler [8] installed in this bypass. The design is identical to the UV system up to the start of the wiggler. Thereafter, we have removed the socalled "THz management chicane", which was provided to alleviate the CSR heat load on the downstream optical cavity mirror. In the absence of an optical cavity, this chicane is unnecessary, and its removal allows space for the additional length of the wiggler footprint. Thereafter, a quadrupole telescope matches the exhaust beam to the original UV energy recovery transport configuration. A schematic is shown in Figure 1; beam envelope functions for this geometry are presented in Figure 2.

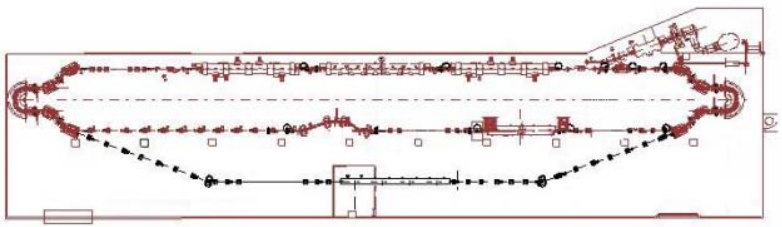

Figure 1: Schematic with long wiggler in "UV bypass".

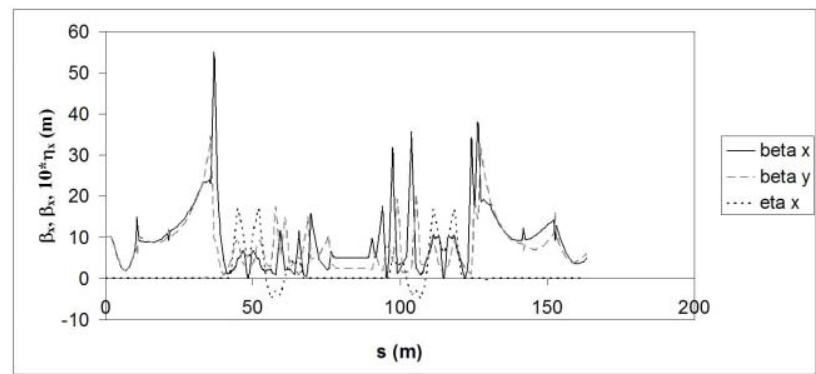

Figure 2: Beam envelope functions.

As the NISUS matched beam envelopes are larger than those in the original UV design, the transverse focusing is more relaxed. This - together with the absence of the chicane - significantly reduces the impact of lattice chromatic aberrations, and we find that the momentum acceptance is significantly larger $(\sim 12 \%$ vs. $\sim 6 \%)$ than in the UV system (which assumed a lower FEL extraction efficiency than is expected in this situation). Figure 3 shows the transverse configuration space and longitudinal phase space after recovery of a $12 \%$ exhaust momentum spread; the beam remains well-defined and regular.

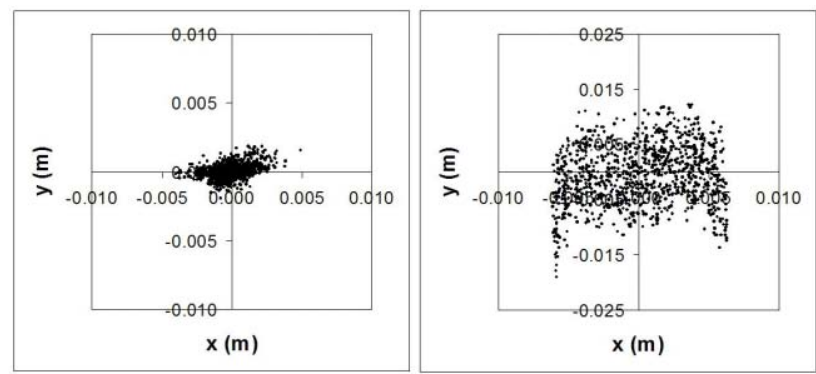

Figure 3: Transverse configuration space and longitudinal phase spaces after energy recovery of exhaust beam from amplifier, assuming $12 \%$ exhaust momentum spread.

The only alternations in the amplifier test configuration from the original UV design are: 1) the transport from the wiggler to the first return dipole (including removal of the $\mathrm{THz}$ chicane), and 2) the use of larger bore quadrupoles in the recovery transport from the backleg axis to the recovery Bates bend, so as to accommodate the larger beam size associated with larger exhaust momentum spread. As noted previously, this bypass beamline has at this date been partially installed under funding for the UV program.

\section{PERFORMANCE CHARACTERIZATION AND DEVELOPMENT}

Amplifier FEL requirements demand that JLab Upgrade drive beam performance be modified from the optimized oscillator operating point. To date, the system regularly delivers to the wiggler a $135 \mathrm{pC}, 115 \mathrm{MeV} / \mathrm{c}$ beam with $10 \mathrm{~mm}$-mrad transverse (both planes) and $90 \mathrm{keV}$-psec longitudinal emittances; the longitudinal is delivered with $\sim 600 \mathrm{keV}$ rms energy spread in a bunch of measured $\sim 160$ fsec rms length. 
The amplifier requires not only a doubled bunch charge $(270 \mathrm{pC})$, but also that the longitudinal match be modified to halve the momentum spread (to $300 \mathrm{keV}$, or $1 / 4 \%$ ) and more than double the bunch length (to $\sim 400 \mathrm{fsec}$ ) while maintaining the transverse emittance. To this end, we have embarked on a machine development program that will, upon completion, provide the appropriate amplifier drive beam. The key elements of this program fall into two categories: injector development and accelerator operation at elevated bunch charge.

\section{Injector Development}

Injector developments must occur in four areas. Firstly, we will increase the gun voltage from $350 \mathrm{kV}$ to $500 \mathrm{kV}$, thereby improving beam performance at higher bunch charge. This activity is now in progress. Secondly, the injector front-end focusing must be strengthened to accommodate both the increased gun voltage and the elevated bunch charge. This is being explored through PARMELA simulation and during initial beam operations studies (see below).

Thirdly, we note that the our specific injector design using a pair of CEBAF-style 5-cell SRF cavities for acceleration of the low energy beam - causes a potentially deleterious deceleration of the low energy beam upon entry into the first cell. This is due to phase slip of the (subrelativistic) beam relative to the cavity RF field while transiting the cavity. Though, notionally, it is desirable to accelerate as rapidly as possible, higher gradients will aggravate this problem. At our usual operating point (350 $\mathrm{kV}$ gun voltage, $\sim 10 \mathrm{MV} / \mathrm{m}$ cavity gradient), the beam is decelerated to approximately $175 \mathrm{keV}$ in the first cell of the first cavity. The effect will be alleviated both by increased gun voltage and by exploration in simulation and operation (see below) of the performance as a function of injection energy. We may find that a lower injection energy - with smaller initial deceleration (before "capture") - will provide better beam performance.

Finally, the injected beam longitudinal aspect ratio must be modified. We note that the longitudinal matching scheme used in this machine performs a $90^{\circ}$ rotation from injection to the wiggler [9]; the injected bunch length is thus imaged as momentum spread, and the injected momentum spread sets the bunch length, at the wiggler. To meet amplifier requirements, we must therefore decrease the bunch length and increase the momentum at injection. Some degree of control of these parameters has already been demonstrated during $10 \mathrm{~kW}$ Upgrade commissioning and operation; exploration of the parameter space - and the longitudinal space charge ("LSC") implications thereof - is underway.

\section{Accelerator Operation at Elevated Bunch Charge}

Two eight-hour accelerator beam study shifts have been devoted to operation at elevated bunch charge. The first shift had as its goal simply the acceleration and recovery of a beam with elevated bunch charge. We raised the charge to $270 \mathrm{pC}$, increased the front-end focusing (both transverse and longitudinal: solenoids and buncher) to compensate the higher charge, and modified the betatron match of the injector to the accelerator. The acceleration cycle was that used for normal operation: injection at 9 $\mathrm{MeV} / \mathrm{c}$, acceleration to $115 \mathrm{MeV} / \mathrm{c}$, recovery to 10.5 $\mathrm{MeV} / \mathrm{c}$. We were able to compress at least a portion of the full energy bunch to $\sim 200$ fsec rms, and fully energy recover the beam. This allowed $\mathrm{CW}$ operation for several minutes at $1 / 2 \mathrm{~mA}$. A measurement of the injected emittance yielded for this un-optimized configuration a value of order $20 \mathrm{~mm}-\mathrm{mrad}$ (normalized).

The second shift began exploring the impact of injection energy and the longitudinal control of the injected beam. We once again raised the bunch charge to $270 \mathrm{pC}$, increased the front end focusing, and altered the injection match. The injected bunch length was shortened and the injected energy spread roughly doubled by appropriate injector phasing. In this configuration, however, we lowered the injection energy to $5.5 \mathrm{MeV} / \mathrm{c}$, accelerated to $110 \mathrm{MeV} / \mathrm{c}$, and recovered to $7 \mathrm{MeV} / \mathrm{c}$. After acceleration, LSC compression of the full momentum spread was observed, with a resulting rms energy spread of $0.4 \%$. The observed bunch length was again, for some portion (but likely not all) of the bunch $\sim 200$ fsec. Given the low injection and recovery energies, we were unable in the available time to establish clean energy recovery. This has been attributed at least in part to the cursory injector setup, the very roughly phased linac and the very preliminary nature of the transverse and longitudinal matching.

Subsequent beam studies will employ higher gun voltage and a more formal (and quantitatively controlled) injector setup. Linac phasing will be performed with care, and both transverse and longitudinal matching performed in detail.

\section{REFERENCES}

[1] G.R. Neil et al, Phys. Rev. Lett., 84, (2000) 662; S. Benson et al, "High Power Operation of the JLAB IR FEL Driver Accelerator", these proceedings.

[2] G.R. Neil et al., op. cit.

[3] S. Benson et al., op. cit.

[4] S.P. Milton, Proc. PAC2001, p.236-40.

[5] H. Loos et al, Proc. EPAC2002, pp. 837-9.

[6] D. Douglas, JLAB-TN-01-051, 26 October 2001.

[7] K. Jordan et al, JLAB-TN-06-045, 8 November 2006.

[8] H. Loos et al, op. cit.

[9] D. Douglas, Proc. LINAC2000. 